\title{
Philonsorbonne
}

$4 \mid 2010$

Année 2009-2010

\section{Imagination et contemplation : Le bon usage de l'imagination selon Pascal}

\section{Tamás PAVLOVITS}

\section{(2) OpenEdition}

\section{$\checkmark$ Journals}

\section{Édition électronique}

URL : https://journals.openedition.org/philonsorbonne/292

DOI : 10.4000/philonsorbonne.292

ISSN : 2270-7336

\section{Éditeur}

Publications de la Sorbonne

\section{Édition imprimée}

Date de publication : 15 mai 2010

Pagination : 123-137

ISBN : 978-2-85944-647-5

ISSN : 1255-183X

\section{Référence électronique}

Tamás PAVLOVITS, «Imagination et contemplation : Le bon usage de l'imagination selon Pascal », Philonsorbonne [En ligne], 4 | 2010, mis en ligne le 02 février 2013, consulté le 11 juin 2021. URL http://journals.openedition.org/philonsorbonne/292 ; DOI : https://doi.org/10.4000/philonsorbonne. 292 


\title{
Imagination et contemplation. Le bon usage de l'imagination selon Pascal
}

\author{
Tamás Pavlovits \\ (Maître de conférences à l'Université de Szeged, Hongrie)
}

L'imagination est une "maîtresse d'erreur et de fausseté » et une « superbe puissance ennemie de la raison ». Les qualifications négatives que Pascal attribue à cette faculté sont dues au fait que l'imagination marque « du même caractère le vrai et le faux » $(44)^{1}$. Autrement dit, la faculté des images en l'homme est indifférente à l'égard de la vérité, car elle produit de la même manière les images qui représentent fidèlement la réalité et celles qui ne sont que des fantasmes. Nombreuses études ont été consacrées à l'analyse du rapport de l'imagination avec la vérité et avec la connaissance dans la pensée pascalienne ${ }^{2}$. Ces commentaires ont montré très justement que l'imagination n'est pas seulement l'ennemie de la raison selon Pascal, mais qu'elle joue un rôle incontournable dans la connaissance et dans la découverte de la vérité, à condition que la raison la dirige.

Dans cette étude, nous allons examiner le fonctionnement de l'imagination dans un contexte particulier et nous réfléchirons sur le rapport qui lie cette faculté au divin selon Pascal. En effet, nous nous bornerons à analyser une seule phrase des Pensées, qui se trouve à la fin du premier paragraphe du fragment intitulé : "Disproportion de l'homme», et qui affirme la chose suivante : "Enfin c'est le plus grand caractère sensible de la toute-puissance de Dieu que notre imagination se perde dans cette pensée » (199). Autrement dit, la réalité de la nature infinie est inimaginable et irreprésentable, et l'échec de l'imagination dans ce processus révèle la

1. Nous citons les Pensées en prenant pour référence l'édition de Pascal : Euvres complètes, éd. Lafuma, coll. «L'Intégrale », Paris, Seuil, 1963. Cette étude a été rédigée avec le soutien du programme de recherche OTKA (F49472).

2. Nous renvoyons au livre de Gérard Ferreyrolles, Les reines du monde, l'imagination et la coutume chez Pascal, Paris, Honoré Champion, 1995 ; et à l'ouvrage de Jean-Pierre Cléro et Gérard Bras, Pascal, Figures de l'imagination, Paris, PUF, 1994. 
toute-puissance de Dieu. La question qui se pose ici est de savoir comment le rapport de l'imagination avec Dieu peut être défini et comment l'imagination rend possible, comme la phrase le suggère, l'expérience de Dieu. Nous essayerons de répondre à cette question en examinant l'usage de l'imagination dans la contemplation, étant donné que l'échec de l'imagination, dont il s'agit là, se produit lors de la contemplation de la nature infinie. Notre thèse consistera à dire que la contemplation définit le bon usage de l'imagination selon Pascal.

\section{La phrase et son contexte}

Voyons d'abord la phrase en question de plus près, et examinons ensuite son contexte. «C'est le plus grand caractère sensible de la toute-puissance de Dieu que notre imagination se perde dans cette pensée », affirme Pascal. Lors de l'analyse de cette phrase, on se heurte à deux problèmes fondamentaux. Le premier concerne l'expression «caractère sensible de la toute-puissance de Dieu». Le mot «sensible» implique que le divin peut devenir l'objet d'une expérience. De quelle expérience s'agit-il ici ? Cette expérience s'effectue par la perte de l'imagination dans la pensée de l'infinité de l'univers. Toutefois, l'échec de l'imagination ne peut pas être une expérience sensible, car lors de cet acte, il ne se produit rien dans l'ordre de la sensibilité. Sans objet sensible, l'expérience à laquelle Pascal fait référence doit être interprétée comme une expérience intérieure que l'homme éprouve au cours de la contemplation de l'infinité de la nature. Cette expérience est attachée au fonctionnement de l'imagination et elle est mise en rapport avec la toute-puissance de Dieu. D'où la deuxième difficulté de l'interprétation de la phrase, relative à son aspect théologique. Elle consiste à savoir pourquoi Pascal parle de l'expérience de la toute-puissance de Dieu à propos de la contemplation de la nature, alors qu'il souligne ailleurs l'impossibilité de déduire l'existence de Dieu à partir de la nature. De nombreux fragments des Pensées insistent sur le fait que le ciel ne prouve pas Dieu et que les auteurs canoniques n'ont jamais utilisé de telles preuves ${ }^{3}$. Dans ces passages, Pascal s'oppose radicalement à la théologie naturelle et rationnelle, de même qu'aux preuves métaphysiques de Dieu. En affirmant que la contemplation rend sensible la toute-puissance de Dieu, cette phrase semble faire exception dans la doctrine pascalienne. Deux questions se posent donc. Comment l'expérience négative de l'échec de l'imagination produit-elle l'expérience du divin ? Comment cette phrase s'accorde-t-elle avec la doctrine pascalienne, qui refuse de déduire l'existence de Dieu de l'observation de la nature?

3. «Et quoi ne dites-vous pas vous-mêmes que le ciel et les oiseaux prouvent Dieu ? Non. Et votre religion ne le dit pas? Non» (4); «C'est une chose admirable que jamais auteur canonique ne s'est servi de la nature pour prouver Dieu» (463). 
Examinons la phrase en question dans son contexte. Après quelques passages rayés, le fragment «Disproportion de l'homme» commence par la phrase suivante : "Que l'homme contemple donc la nature entière dans sa haute et pleine majesté ». Il s'agit donc de décrire le processus de la contemplation conduisant à l'échec de l'imagination. L'objet de la contemplation est la totalité de la nature. Le processus part des choses sensibles, qui entourent l'homme, pour s'élever à la contemplation des choses célestes.

Que l'homme «éloigne sa vue des objets bas qui l'environnent. Qu'il regarde cette éclatante lumière mise comme une lampe éternelle pour éclairer l'univers, que la terre lui paraisse comme un point au prix du vaste tour que cet astre décrit, et qu'il s'étonne de ce que ce vaste tour lui-même n'est qu'une pointe très délicate à l'égard de celui que ces astres, qui roulent dans le firmament, embrassent ».

Dans un premier temps, la contemplation s'effectue de manière sensible, à savoir par les yeux. Le tour des étoiles désigne la limite de la vue mais cette limite est loin d'être celle de la nature. C'est pourquoi la contemplation doit se poursuivre par l'imagination: "Mais si notre vue s'arrête là que l'imagination passe outre, elle se lassera plutôt de concevoir que la nature de fournir ».

Si la vue est limitée, l'imagination ne l'est pas. Son caractère lui permet d'approcher facilement de l'objet ultime de la contemplation : Pascal affirme dans une de ses lettres, que «l'imagination a cela de propre qu'elle produit avec aussi peu de peine et de temps les plus grandes choses que les petites $»^{4}$. L'imagination joue donc un rôle indispensable dans la contemplation de la nature, parce qu'elle est à même de représenter les parties qui s'étendent au-delà des limites de la visibilité.

Lors de la contemplation de la nature, l'imagination s'efforce de la représenter telle qu'elle est. Il semble cependant qu'il y ait une autre faculté qui intervienne dans la contemplation : celle de conception. Pascal souligne qu'aucune idée intelligible n'approche la réalité de la nature et que «nous avons beau enfler nos conceptions au-delà des espaces imaginables, nous n'enfantons que des atomes au prix de la réalité des choses ». En distinguant entre l'imagination et la conception, Pascal semble suivre Descartes. Selon la Sixième méditation, la conception demande moins d'effort que l'imagination et s'étend au-delà de celle-ci. Alors que l'imagination ne peut représenter un chiliogone que confusément, par la conception pure on peut facilement en déduire des vérités indubitables 5 . Dans le fragment 199, Pascal affirme que même si l'on essaie de pousser les limites des tentatives représentatives au-delà de l'imagination, l'idée produite ne sera jamais adéquate à la réalité qu'elle vise à représenter. La distinction entre

4. Lettre de Pascal au Père Noël, in Pascal, Euvres complètes, éd. J. Mesnard, t. II, Paris, Desclée de Brouwer, 1970, p. 552.

5. Cf. Sixième méditation, AT, t. IX, p. 57-58. 
l'imagination et la conception sert à mettre en évidence la faillite de l'imagination au cours du processus contemplatif. La raison prend conscience de l'échec de l'imagination en constatant que l'image produite par celle-ci n'est nullement adéquate au but ultime de la représentation, notamment à la totalité de la nature.

La présentation du processus de la contemplation se conclut par ces deux phrases très célèbres :

C'est une sphère infinie dont le centre est partout et la circonférence nulle part. Enfin c'est le plus grand caractère sensible de la toute-puissance de Dieu que notre imagination se perde dans cette pensée.

La métaphore de la sphère infinie présente une image inimaginable et une idée inconcevable de l'univers, en soulignant encore une fois l'échec des facultés représentatives de l'homme en face de la nature infinie.

Après avoir montré comment l'imagination se perd dans la contemplation de l'immensité de l'univers, Pascal présente le même processus contemplatif en sens inverse: vers les éléments infimes de la nature. En dessinant l'image des univers infimes, des firmaments, des planètes et des terres dans «l'enceinte de ce raccourci d'atome» qu'une goutte de sang d'un ciron représente, il fait de nouveau travailler l'imagination. Le résultat de la contemplation de la petitesse de la nature est le même que celui de la considération de sa grandeur: l'imagination s'y perd. «Il se perdra dans ces merveilles aussi étonnantes dans leur petitesse que les autres par leur étendue », dit Pascal. La nature est donc irreprésentable et sa contemplation, dans son immensité aussi bien que dans sa petitesse, conduit à l'échec de l'imagination.

Le processus de la contemplation rend la phrase présentant la relation entre l'imagination et le divin encore plus énigmatique. Dans le fragment 199, Pascal parle très peu de Dieu, étant donné que ce fragment développe des arguments fondés sur la lumière naturelle et qu'il vise à mettre en évidence la disproportion entre l'homme et la nature infinie. Par conséquent, toutes les considérations concernant le surnaturel sont écartées, excepté quelques-unes. L'exemple de la contemplation semble servir à prouver que l'homme, étant incapable de forger une représentation authentique de la nature, ne peut pas la connaître et que cette incapacité lui rend impossible la connaissance de Dieu par des moyens naturels ${ }^{6}$. «Manque d'avoir contemplé ces infinis les hommes se sont portés témérairement à la recherche de la nature comme s'ils avaient quelque proportion avec elle », écrit Pascal un peu plus bas. Pourquoi parle-t-il donc du caractère sensible de Dieu à propos de l'échec de l'imagination, alors que le caractère inconcevable et irreprésentable de la nature rend évidente l'impossibilité de forger des preuves rationnelles de l'existence de Dieu?

6. Nous trouvons la même conception dans le fragment 418 , où l'infinité de la nature crée une rupture infranchissable entre le fini et Dieu. 
Cela revient à nous demander quel rapport peut s'établir, selon Pascal, entre l'imagination et le divin au cours de la contemplation. Afin de définir ce rapport, il nous faut comparer la contemplation pascalienne avec la contemplation traditionnelle.

\section{La contemplation traditionnelle}

À défaut de donner une présentation exhaustive de la littérature philosophique traitant de la contemplation, examinons quelques passages importants afin de dégager les traits caractéristiques de cet acte intellectuel. La contemplation a donné lieu à une littérature très riche dans la tradition antique et médiévale. Sa théorie est élaborée pour la première fois par Platon et Aristote, que Festugière nomme «les théoriciens et les apôtres de la contemplation $»^{7}$. La contemplation joue un rôle important dans la pensée stoïcienne et néo-platonicienne. Au Moyen Âge, les penseurs chrétiens conservent les traits caractéristiques de la contemplation antique et ne les modifient que légèrement. Ce sont surtout les mystiques qui en font la théorie. La théorie et la pratique de la contemplation se fondent sur un arrière-plan cosmologique, ontologique et théologique où ces trois doctrines trouvent un accord parfait. L'objet principal de la contemplation, selon les théories antiques, est l'ordre cosmique à travers lequel le divin se donne à voir. D'après son étymologie, le mot grec «theoría » (contemplation) a une parenté avec le mot «oráo » qui signifie voir ou observer. Pour contempler il faut d'abord voir. La contemplation passe toujours du sensible à l'intelligible et assure, de cette manière, l'élévation du naturel au surnaturel. Platon, dans le Timée, présente ce processus de la manière suivante :

Pour nous, nous dirions que la cause en vertu de laquelle le dieu a inventé la vision et nous en a fait présent est la suivante et toujours la même. Ayant contemplé les mouvements périodiques de l'intelligence dans le Ciel, nous les utiliserons, en les transportant aux mouvements de notre propre pensée, lesquels sont de même nature, mais troublés, alors que les mouvements célestes ne connaissent pas de trouble. Ayant étudié à fond ces mouvements célestes, participant à la rectitude naturelle des raisonnements, imitant les mouvements divins qui ne comportent absolument aucune erreur, nous pourrions stabiliser les nôtres, qui ne cessent point errer ${ }^{8}$.

Les objets de la contemplation sont les mouvements célestes qui représentent par leur caractère ordonné l'intelligence divine. L'homme peut voir ces mouvements et en comprenant leur ordre peut rendre sa pensée aussi ordonnée qu'ils le sont. Ainsi, lors de la contemplation de l'ordre céleste et cosmique, l'homme devient semblable à Dieu et parvient à imiter la pensée divine. L'imitation ne devient parfaite que par l'acte de la nóésis

7. A. J. Festugière, Contemplation et vie contemplative selon Platon, Paris, Vrin, 1936, p. 18.

8. Platon, Timée, 47 b-c, traduction d'A. Rivaud, Paris, Les Belles Lettres. 
(intellection) qui mène au-delà de la contemplation. Par conséquent, la theoría « dit un sentiment de présence, un contact avec l'Être saisi dans son existence. Cette saisie dépasse et le langage et l'intellection. L'objet vu est au-delà de l'ousía. Il est ineffable. Il ne se laisse circonscrire en nulle définition », résume Festugière?

Pour Aristote aussi, la contemplation conduit la philosophie à son achèvement. Toutefois, chez lui, la contemplation est l'activité principale du Premier Moteur. Dieu se contemple en se pensant soi-même. Sa pensée est la nóésis perpétuelle dans laquelle le sujet et l'objet de la pensée sont uniques $^{10}$. L'homme, dans de rares moments de sa vie, peut s'élever par la contemplation à cet état. La contemplation, de même que chez Platon, met en œuvre le noûs qui est la partie divine et la partie fondamentale de l'être de l'homme $^{11}$. Par conséquent, la contemplation conduit au moment où l'esprit humain s'unit avec l'esprit divin et où l'homme atteint le vrai bonheur :

Le bonheur est donc coextensif à la contemplation, et plus on possède la faculté de contempler [le noûs], plus on est heureux, heureux non pas par accident, mais en vertu de la contemplation même, car cette dernière est par elle-même d'un grand prix. Il en résulte que le bonheur ne saurait être qu'une forme de la contemplation ${ }^{12}$.

Nous voyons chez les grands théoriciens de la contemplation que cet acte signifie l'achèvement de la pensée philosophique, qu'il va du sensible à l'intelligible et qu'il rend possible l'élévation jusqu'au divin. La contemplation s'achève par la saisie de l'Un qui correspond au bonheur suprême.

Dans la tradition chrétienne, la contemplation revêt le même sens. Chez les mystiques, elle joue un rôle important dans l'unio mystica. La contemplation permet de s'élever du naturel au surnaturel et de parvenir à la vision de Dieu. Maître Eckhart souligne qu'au cours de ce processus la contemplation conduit au-delà de la contemplation même :

Mais bien que nous prenions congé par-là de tout le monde fini et nous engagions sur la voie de la vérité, nous ne sommes cependant pas pleinement bienheureux, même si nous contemplons la vérité divine. Aussi longtemps que nous nous en tenons à la contemplation nous ne sommes pas encore en ce que nous contemplons, aussi longtemps qu'un quelque chose est l'objet de notre attention nous ne sommes pas Un dans l'Un. Car là où il n'y a rien qu'une chose, on ne voit rien! D'où il suit qu'on ne peut voir Dieu qu'en étant aveugle, le connaître qu'en étant ignorant et ne le comprendre qu'en étant déraisonnable ${ }^{13}$.

9. A. J. Festugière, op. cit., p. 5.

10. Cf. les fameuses pages de la Métaphysique, livre $\Lambda, 9,1074 b$ 34-1075 a 5 .

11. Cf. Ethique à Nicomaque, $\mathrm{X}, 7,1177$ b 25-1178 a 8 .

12. Ibid., X, 9, $1178 b$, trad. J. Tricot.

13. Des obstacles de la vraie spiritualité, in Euvres de Maître Eckhart, trad. P. Petit, Paris,

Gallimard, 1987, p. 294. 
Sans citer d'autres exemples de la contemplation chrétienne (comme ceux de saint Augustin ou du Pseudo-Denys l'Aréopagite) nous pouvons constater que l'enjeu et la pratique de la contemplation sont les mêmes au Moyen Âge et dans l'Antiquité.

Dans ces théories, toutefois, l'imagination ne semble pas intervenir dans le processus contemplatif. Afin de préciser son rôle dans la contemplation médiévale, il faut se référer à l'œuvre de Richard de Saint-Victor, au Benjamin minor, De la préparation de l'âme à la contemplation. Dans cette œuvre, Richard distingue six degrés de la contemplation :

Le premier est dans l'imagination et selon l'imagination seule. Le second est dans l'imagination et selon la raison. Le troisième est dans la raison selon l'imagination. Le quatrième est dans la raison et selon la raison. Le cinquième est au-dessus de la raison, mais il ne la laisse pas de côté. Le sixième est audessus de la raison, et il semble la laisser de côté ${ }^{14}$.

Au premier degré, l'homme contemple la beauté de la nature sans réfléchir. Au deuxième, il cherche à l'aide de son esprit la raison des choses et des processus naturels. Comme Richard l'explique : «dans le premier genre $[\ldots]$ ce sont les choses mêmes, dans le second ce sont surtout leur raison, leur ordre, leur disposition et la cause de chaque chose, son mode et son utilité que nous scrutons, que nous observons par la spéculation, que nous admirons $»^{15}$. Dans ces deux cas, l'imagination se confond visiblement avec la sensibilité. Le troisième degré de la contemplation consiste à s'élever du sensible à l'intelligible à travers les similitudes entre le naturel et le surnaturel et à travers les symboles qui se fondent sur ces similitudes. Le quatrième degré est celui où la raison, après s'être détachée du sensible, contemple uniquement les formes intelligibles. Le cinquième degré s'accomplit déjà par l'intelligence et se fonde sur la révélation divine ; le sixième degré correspond à l'achèvement de la contemplation, à savoir à l'union avec le divin.

Dans ce processus, ce sont les deuxième et troisième degrés qui nous importent le plus, parce qu'ils révèlent le rôle de l'imagination dans la contemplation. Comme chez les Grecs, la contemplation part de la nature sensible. Cette démarche explique l'importance de l'imagination qui signifie, dans ce cas-là, la sensibilité (la faculté des images). Au troisième degré, où il s'agit de quitter l'ordre du sensible, l'imagination fonctionne sous la direction de la raison. L'élévation du sensible à l'intelligible s'effectue grâce à la similitude entre les choses naturelles et les êtres surnaturels : l'imagination rend présentes les choses sensibles et la raison y découvre des symboles, c'est-à-dire des traces du surnaturel au sein du naturel. Cette troisième forme de la contemplation s'effectue dans la raison selon l'imagination et définit précisément le rôle de l'imagination dans cet acte.

14. In Théologiens et mystiques au Moyen Âge, éd. A. Michel, Paris, Gallimard, 1997, p. 358. 15. Ibid., p. 359. 
Les traits caractéristiques de la contemplation pré-moderne sont donc les suivants : elle assure l'élévation du sensible à l'intelligible, elle se fonde sur le fonctionnement simultané de la sensibilité, de l'imagination et de la raison, elle aboutit dans l'intellection qui correspond à la saisie de l'Un, à la vision de Dieu et à l'unio mystica, et, enfin, elle s'achève par l'atteinte du bonheur suprême. Il est nécessaire de souligner aussi que le passage du naturel au surnaturel s'effectue par la contemplation de l'ordre du monde et par la reconnaissance de la similitude entre la nature sensible et la nature divine.

\section{La contemplation pascalienne}

Revenons maintenant à la contemplation pascalienne. Vincent Carraud a fait une analyse approfondie du fragment 199 des Pensées du point de vue de la contemplation, concluant que la démarche intellectuelle mise en scène dans ce fragment doit être qualifiée d'anti-contemplation. Pascal, écrit Vincent Carraud, « opère la destruction du concept de contemplation, tel que la tradition l'avait élaboré par des enrichissements successifs. [...] Tout se passe donc comme si le concept de contemplation, dont Pascal hérite de la tradition chrétienne, gouvernait le $\S 199$, alors que, derrière l'emploi rhétorique du mot, c'est de la ruine du concept qu'il s'agira, et de la fin d'une tradition spirituelle particulièrement riche ${ }^{16}$. D'après l'interprète, la contemplation pascalienne doit être considérée comme une anticontemplation car au lieu d'être la contemplation de Dieu, elle se réduit à la contemplation de la nature, et qu'au lieu d'aboutir à l'union avec le divin et à la béatitude, elle se termine par l'effroi. Notre thèse diffère de celle de Vincent Carraud, dans la mesure où nous ne voyons pas une opposition aussi forte entre la contemplation traditionnelle et la contemplation pascalienne. Pascal ne détruit pas le concept de contemplation, mais le transforme. Essayons de comprendre comment.

Les étapes principales de la démarche contemplative pascalienne sont identiques à celles de la contemplation traditionnelle. Nous avons vu dans le fragment 199 comment la contemplation part du sensible, s'élève à l'intelligible par l'imagination et par l'intellection, pour parvenir enfin au caractère sensible de la toute-puissance de Dieu. Cependant, dans leur contenu, le processus traditionnel et le processus pascalien diffèrent visiblement. Afin de comprendre en quoi consiste cette différence, nous devons comparer leur objet. L'objet principal de la contemplation pascalienne est, comme chez les Grecs et chez les médiévaux, la nature. Toutefois, cet objet diffère fortement de l'objet de la contemplation antique et médiévale, étant donné que la nature, selon Pascal, n'est pas ordonnée de

16. Vincent Carraud, Pascal et la philosophie, Paris, PUF, coll. «Epiméthée », 1992, p. 404 et 406 . 
la même manière que le cosmos pré-copernicien. Il est possible d'analyser la différence de deux manières : cosmologique et ontologique. Le cosmos ou la création visible assuraient un passage immédiat du sensible à l'intelligible, grâce à l'ordre qu'ils ont inclus. Alors que l'ordre cosmique était donné à voir par les éléments sensibles (planètes, étoiles), son essence consistait en l'arrangement de leur mouvement défini par des règles intelligibles. À travers ces règles ou lois cosmiques, l'ordre sensible faisait référence à l'existence d'un principe ordonnateur, c'est-à-dire à Dieu même dont la pensée s'exprimait par ces règles. L'arrière-plan cosmologique rendait ainsi possible l'élévation de la pensée humaine au divin par la contemplation des mouvements célestes. Outre l'explication cosmologique, il y avait une raison ontologique qui fondait également l'élévation contemplative. L'ordre cosmique, en étant identique à la forme du monde, était en même temps un ordre ontologique. Comme tel, il constituait l'unité dans le multiple et assurait la présence de l'intelligible dans le sensible. La contemplation de l'ordre du cosmos revenait ainsi à la contemplation de la forme et de l'unité du monde. L'ordre, en tant que manifestation de l'Un dans la nature, permettait à l'homme son élévation vers l'Un.

Chez Pascal, l'infinité prive l'univers de son ordre visible. Il s'ensuit que la contemplation ne peut pas s'élever du sensible à l'intelligible de manière immédiate. La vision ne peut pas saisir l'unité de la nature, car l'infinité l'en empêche. Son objet lui échappant, la vision doit être complétée par l'imagination. Néanmoins, l'imagination n'est toujours pas à même d'assurer le passage immédiat du sensible à l'intelligible, c'est pourquoi elle vise d'abord à représenter la nature réelle dans sa totalité. Ici, nous pouvons saisir la différence essentielle entre la contemplation traditionnelle et la contemplation pascalienne. Selon la cosmologie antique et médiévale, le cosmos était identique au monde visible dans la mesure où le firmament était la limite du cosmos. Par conséquent, la représentation de la totalité du monde s'effectuait immédiatement par la vision. Par contre, dans l'univers post-copernicien, il y a un écart infini entre le monde visible et l'univers réel. Lors de la contemplation de la nature, c'est à l'imagination de franchir cet écart en essayant de forger une représentation intelligible de la totalité de la nature à partir de ses parties sensibles. Or, comme le fragment 199 le souligne, l'imagination est aussi impuissante à saisir l'objet à contempler que la vision, et subit un échec en essayant de le représenter. Elle se perd dans cette pensée.

La mise en évidence de la différence entre la contemplation traditionnelle et pascalienne nous fait mieux comprendre la phrase en question. Il va de soi que Pascal parle de Dieu à la fin de la présentation du processus contemplatif, étant donné que cet acte s'achève traditionnellement par la vision de Dieu. Ce faisant, il reste fidèle à la tradition, mais souligne en même temps que le sens de la contemplation a profondément changé après la révolution copernicienne. Pascal redéfinit entièrement la contemplation. Chez lui, la contemplation de la nature infinie n'aboutit pas à la vision de Dieu, ni à l'unio mystica. La raison en est que les facultés 
humaines sont incapables de représenter la nature et, par conséquent, elles ne sont pas à même de saisir son unité par sa forme. La contemplation pascalienne reste prisonnière de la représentation. Le processus de représentation est infini : l'imagination est à la recherche de l'objet ultime de la contemplation sans pouvoir le saisir. L'échec de la représentation implique donc que l'Un ne peut pas être saisi dans le multiple et que l'homme ne peut pas s'élever par la nature jusqu'à Dieu. La phrase vise à permettre de tirer cette conclusion.

Cette interprétation n'épuise pas cependant la signification de la phrase. S'agirait-il seulement de constater que la contemplation de la nature infinie ne permet ni de saisir l'existence de Dieu, ni de s'unir à lui ? Dans ce cas-là, nous serions obligés d'accepter l'analyse de Vincent Carraud et de dire avec lui que la contemplation pascalienne est une anti-contemplation. Toutefois, la forme syntaxique de la phrase suggère qu'elle a une signification plus complexe. Elle ne se réduit pas à la constatation d'un fait négatif, en disant que la contemplation de la nature infinie ne conduit guère à Dieu, mais affirme quelque chose de positif, à savoir que l'échec de l'imagination dans la contemplation est le caractère sensible de la toute-puissance de Dieu. Afin de comprendre cette affirmation, nous devons revenir à l'examen de l'imagination.

\section{L'imagination et le divin}

La question est de savoir si l'imagination peut fonder un rapport authentique au surnaturel selon Pascal. Dans deux autres fragments des Pensées, il aborde cette question, notamment dans les fragments 432 et 551. Dans les deux cas, il s'agit de la capacité de l'imagination à agrandir ou à amoindrir les choses sans peine. Cette capacité concerne le rapport naturel de l'homme à Dieu. "L'imagination grossit les petits objets jusqu'à en remplir notre âme par une estimation fantasque, et par une insolence téméraire elle amoindrit les grandes jusqu'à sa mesure, comme en parlant de Dieu », écrit Pascal dans le fragment 551. En grandissant et en amoindrissant les objets l'imagination relie des distances immenses. Néanmoins quand elle s'applique au divin, elle commet une faute inexcusable. Ne pouvant représenter Dieu qu'à partir des choses naturelles et finies, elle réduit le divin à la mesure de l'humain et supprime la rupture radicale entre le naturel et le surnaturel. Par conséquent, il est illusoire de fonder notre rapport au divin sur l'activité de l'imagination. Dans le fragment 432, il s'agit de la même faute, commise à propos du temps et de l'éternité : "Notre imagination nous grossit si fort le temps présent à force d'y faire des réflexions continuelles, et amoindrit tellement l'éternité, manque d'y faire réflexion, que nous faisons de l'éternité un néant, et du néant une éternité ; et tout cela a ses racines si vives en nous que toute notre raison ne nous en peut défendre ». L'activité de l'imagination crée encore des illusions lorsqu'elle 
prétend représenter l'éternité. Suite à son usage inadéquat, l'éternité divine devient un néant et le temps humain devient l'éternité ${ }^{17}$. Cela revient à dire que le surnaturel et le naturel se trouvent représentés selon la même mesure ou bien que l'imagination forge des représentations fausses de l'irreprésentable. La source de cette erreur consiste en l'absence de réflexion sur la nature de l'objet représenté. En faisant ses opérations, l'imagination ne fait pas cette réflexion, c'est à la raison de l'effectuer. L'imagination s'avère donc puissance trompeuse quand l'homme veut créer un rapport à Dieu à l'aide de cette faculté et en négligeant la raison.

Pascal n'est pas le seul à souligner qu'il faut se méfier d'imaginer Dieu à partir des choses finies. Descartes lui-même insiste sur ce point dans une lettre à Mersenne en parlant de la création des vérités éternelles. Il est temps, écrit-il, «que le monde s'accoutume de parler de Dieu plus dignement, ce me semble, que n'en parle le vulgaire, qui l'imagine presque toujours ainsi qu'une chose finie $»^{18}$.

Toutefois, Pascal ne rejette pas entièrement la possibilité de maîtriser l'imagination par la raison, et d'en faire un bon usage dans son rapport au divin. Selon notre thèse, le processus de la contemplation définit, dans le fragment 199, le bon usage de l'imagination lorsque l'homme veut se détourner du naturel pour se tourner vers le surnaturel. De quoi s'agit-il ? Pendant tout le processus, l'imagination est gouvernée par la raison et est forcée de représenter la nature infinie. Cette contrainte, comme nous l'avons montré, rend impossible de passer de la nature sensible à Dieu, étant donné que l'imagination ne peut pas parcourir l'infini, ni s'élever à l'intelligible pur. Afin de saisir l'objet de la contemplation, notamment la nature infinie, l'imagination doit mettre en œuvre toute sa force. Ce faisant, elle est obligée de constater que l'infini échappe à sa capacité représentative. L'imagination est d'abord poussée à l'extrême, ensuite elle fait l'expérience de son échec. L'échec de l'imagination provoque une expérience intérieure mais opposée à l'expérience ultime de la contemplation traditionnelle. Au lieu de trouver le point absolu, cette expérience consiste en la perte de tous les points de repère ; au lieu de parvenir à l'Un, l'échec de l'imagination consiste à se perdre dans l'infinité irreprésentable.

Bien que l'expérience que la perte de l'imagination provoque soit une expérience négative, elle n'en est pas moins une expérience. Sans aucun doute, elle n'est pas l'expérience de la présence de Dieu : elle est l'expérience de son absence. L'imagination gouvernée par la raison dans la contemplation, loin de forger une fausse image de Dieu et de l'éternité, provoque l'expérience de l'absence de Dieu dans la nature. La contemplation dans le fragment 199 parvient donc, à travers le bon usage de l'imagination, à l'expérience de Dieu, typique de Pascal, celle du Dieu caché.

17. Dans ce deuxième cas, il s'agit de l'oubli de la mort. Ce motif est très fréquent dans les Pensées.

18. Lettre à Mersenne, le 15 avril 1630. 


\section{Le sublime et le caractère sensible de la toute-puissance de Dieu}

L'échec de l'imagination lors de la représentation de la nature infinie semble produire en l'homme une expérience semblable à celle que Kant nomme, dans la Critique de la faculté de juger, le «sentiment du sublime». En effet, selon la définition kantienne : «est sublime ce en comparaison de quoi tout le reste est petit $\gg^{19}$. Par conséquent, c'est la nature infinie qui doit être qualifiée de sublime : «La nature est donc sublime dans ceux de ses phénomènes dont l'intuition implique l'idée de son infinité ${ }^{20}$, écrit Kant. En même temps, il souligne que le sublime n'est pas la qualité d'un objet, mais qu'il est un sentiment ou une émotion intérieure. Cette émotion s'attache à un certain usage représentatif de l'imagination. Le sentiment du sublime est provoqué par l'inadéquation entre l'imagination, comme faculté sensible, et la raison, comme faculté suprasensible, lorsque la première effectue une évaluation esthétique de la grandeur. L'évaluation esthétique de la grandeur exprime la tentative de l'imagination pour représenter la totalité de l'univers. Or, cette tentative, comme Pascal lui-même le signale clairement, s'effectue par une progression qui tend vers l'infini. L'imagination, étant une faculté sensible, ne parvient jamais à représenter la totalité de la nature infinie, parce que la sensibilité l'attache au fini qui est en opposition radicale avec l'infini. L'inadéquation entre l'imagination et la raison résulte de la faiblesse de la première qui s'oppose à l'exigence de la deuxième faculté. Kant écrit :

L'effort extrême de l'imagination pour présenter une unité à l'évaluation de la grandeur se réfère à quelque chose d'absolument grand ; par conséquent, considérer l'absolument grand comme seule mesure suprême des grandeurs, c'est se référer également à la loi de la raison. Donc la perception interne de l'inadéquation de tout critère sensible par rapport à l'évaluation de la grandeur par la raison s'accorde aux lois de celle-ci et suscite un déplaisir qui provoque en nous le sentiment de notre destination suprasensible, pour laquelle découvrir que tout critère de la sensibilité est inadéquat par rapport aux idées de la raison est conforme à une finalité et provoque donc du plaisirir .

Un phénomène analogue se retrouve chez Pascal : la mise en évidence de l'incapacité de l'imagination à forger une représentation adéquate de l'univers infini provoque à la fin de la contemplation quelque chose comme le sentiment du sublime. Pour Pascal, ce sentiment est le « caractère sensible de la toute-puissance de Dieu », en admettant qu'il ne s'agisse pas de l'expérience de la présence mais de l'absence de Dieu. Kant donne une analyse très précise de ce sentiment intérieur que nous devons prendre en considération afin d'éclairer la nature de la contemplation pascalienne.

19. Kant, Critique de la faculté de juger, trad. Marc B. de Launay, Paris, Gallimard, 1985, p. 189.

20. Ibid., p. 195

21. Ibid., p. 199. 
Comme le passage cité plus haut le soulignait déjà, le sentiment du sublime est un sentiment double qui se compose du plaisir et du déplaisir. Kant explique qu'en parvenant au sentiment du sublime, l'esprit n'est pas dans un état contemplatif calme mais «se sent mis en mouvement». Or ce mouvement «peut-être comparé à un ébranlement, c'est-à-dire à une rapide alternance de répulsion et d'attrait provoqués par le même objet ${ }^{22}$. L'objet en question est bien entendu la nature infinie qui est attirante et répugnante à la fois :

Ce qui est excessif à l'imagination (c'est-à-dire ce à quoi elle est conduite dans l'appréhension ou l'intuition) est en quelque sorte un abîme où elle est crainte elle-même de se perdre; en revanche, pour l'idée rationnelle du suprasensible, produire pareil effort d'imagination n'est pas excessif, mais conforme à la loi ; c'est donc ce qui est à son tour attirant dans la mesure exacte où c'était repoussant pour la seule sensibilitée ${ }^{23}$.

De l'inadéquation entre l'imagination et la raison au cours de l'évaluation esthétique de la grandeur, résulte donc un sentiment complexe qui se compose de deux sentiments contraires. Pour comprendre la nature véritable du sentiment pascalien de la toute-puissance de Dieu, il faut observer qu'il se compose également de deux sentiments opposés.

Lorsque Vincent Carraud écrit que la contemplation pascalienne est une anti-contemplation, il fonde son opinion (entre autres) sur le fait que, contrairement à la contemplation traditionnelle conduisant à la béatitude, elle provoque l'effroi. Il va de soi que la perte de l'imagination dans la contemplation de la nature infinie ne procure jamais le bonheur. Mais il faut voir en même temps qu'elle ne provoque pas seulement l'effroi, mais aussi le sentiment de l'admiration. Pascal affirme plusieurs fois que l'infinité est admirable aussi bien qu'effroyable. Dans l'Esprit géométrique, il ne parle que de l'admiration à propos du double infini : «Voilà l'admirable rapport que la nature a mis entre ces choses, et les deux merveilleuses infinités, qu'elle a proposées aux hommes, non pas à concevoir, mais à admirer ${ }^{24}$. Dans ce passage, l'admiration de l'infini n'est pas mise en rapport avec sa contemplation. Par contre, dans le fragment 199, le rapport est évident : "Qui se considérera de la sorte s'effraiera de soi-même et se considérant soutenu dans la masse que la nature lui a donnée entre ces deux abîmes de l'infini et du néant, il tremblera dans la vue de ces merveilles et je crois que sa curiosité se changeant en admiration il sera plus disposé à les contempler en silence qu'à les rechercher avec présomption ».

Il faut ajouter à ces considérations qu'au XVII ${ }^{\mathrm{e}}$ siècle, l'adjectif «effroyable» signifiait également «admirable». Selon le dictionnaire Furetière, ce mot «se dit aussi de ce qui est prodigieux, qui surprend, qui

24. Pascal, Euvres complètes, éd. J. Mesnard, t. III, Paris, Desclée de Brouwer, 1991, p. 410. 
cause de l'admiration » et il en donne un exemple étonnant pour notre propos : «l'étendue des cieux est effroyable». Quand Pascal écrit que «le silence éternel de ces espaces infinis m'effraie» (201), il affirme donc que la contemplation de la nature infinie provoque l'effroi et l'admiration en même temps. Cela nous permet de conclure la description du processus contemplatif du fragment 199: l'échec de l'imagination lors de la tentative pour représenter l'univers infini provoque un sentiment double (un plaisir et un déplaisir, une joie et une horreur, l'admiration et l'effroi, l'attirance et la répugnance) qui s'attache au même objet, à savoir à la nature infinie.

Pascal va cependant plus loin que Kant : il suggère que ce sentiment double est en rapport avec l'ordre du sacré. Philippe Sellier a déjà proposé de voir dans le double sentiment de l'effroi et de l'admiration provoqué par l'infini le sentiment du sacré. Il cite saint Augustin afin de montrer que l'évêque d'Hippone fait lui-même un rapprochement entre l'admiration et l'effroi à propos de la contemplation et que la contemplation pleine d'effroi et d'admiration du monde est la contemplation de Dieu même ${ }^{25}$. Toutefois, il semble que la contemplation ne conduise pas au même sentiment du sacré chez Pascal et chez saint Augustin. L'abîme de la représentation dans lequel l'imagination tombe lors de la contemplation consiste à perdre tous les points de repère et implique un sentiment de chute que la contemplation du cosmos pré-moderne n'effectue jamais. C'est pourquoi nous disons que le double sentiment de l'effroi et de l'admiration n'est pas une expérience positive mais une expérience négative de Dieu. En se perdant dans la pensée de l'infinité de l'univers, l'imagination fait l'expérience de l'absence de Dieu dans la nature. Autrement dit, sa perte provoque l'expérience naturelle de Dieu qui reste, en dépit de tous les efforts naturels, profondément caché.

Le rapprochement entre le résultat de la contemplation pascalienne et le sublime kantien nous a permis de donner à la phrase du fragment 199 une interprétation cohérente. La perte de l'imagination lors de la contemplation de la nature infinie cause une expérience intérieure qui semble correspondre à l'émotion du sublime. «Le caractère sensible » et naturel de Dieu est ainsi un sentiment que l'homme éprouve si son imagination est bien conduite en contemplant la nature. Ce sentiment est double : il se compose d'effroi et d'admiration. La présence simultanée de l'admiration et de l'effroi dans ce sentiment permet de l'interpréter comme le sentiment du sacré. Toutefois, le sacré ne peut y être présent de la même manière que dans la béatitude éprouvée à l'issue de la contemplation traditionnelle. Dieu n'y est présent que par son absence. La référence à la toute-puissance de Dieu dans le fragment 199 prouve néanmoins que la contemplation pascalienne n'est pas

25. «Devant la splendeur du monde il [l'homme] éprouve ce sentiment de fascination et d'effroi que provoque la rencontre du sacré. [...] Mais l'existence du même sentiment dans le texte sur les deux infinis et le fait que l'auteur considère qu'il s'agit là d'une impression commune à tout spectateur conscient du monde nous orientent vers la solution que nous venons d'évoquer : le sens du sacré. Et nous aboutissons bientôt à une quasi certitude, lorsque nous rencontrons dans saint Augustin exactement les mêmes mouvements » (Philippe Sellier, Pascal et saint Augustin, Paris, Albin Michel, 1995, p. 363 et 364). 
une anti-contemplation: elle demeure contemplation quoique son sens change profondément. Le bon usage de l'imagination dans la contemplation de la nature ne conduit pas à l'ordre du cœur, où la certitude de Dieu se révèle, mais mène au moins à l'expérience existentielle de l'absence de Dieu dans la nature. 